\title{
The harmonic and sideband structure of the kilohertz quasi-periodic oscillations in Sco X-1
}

\author{
Mariano Méndez ${ }^{1,2 \star}$ and Michiel van der Klis ${ }^{1}$ \\ ${ }^{1}$ Astronomical Institute 'Anton Pannekoek', University of Amsterdam and Center for High-Energy Astrophysics, Kruislaan 403, NL-1098 SJ Amsterdam, \\ the Netherlands \\ ${ }^{2}$ Facultad de Ciencias Astronómicas y Geofísicas, Universidad Nacional de La Plata, Paseo del Bosque S/N, 1900 La Plata, Argentina
}

Accepted 2000 June 20; Received 2000 June 20; in original form 2000 February 28

\begin{abstract}
A B S T R A C T
We use data from the Rossi X-ray Timing Explorer to search for harmonics and sidebands of the two simultaneous kilohertz quasi-periodic oscillations (kHz QPOs) in Sco X-1. We do not detect any of these harmonics or sidebands, with 95 per cent confidence upper limits to their power between $\sim 1$ and $\sim 10$ per cent of the power of the upper $\mathrm{kHz}$ QPO. The oscillations produced at these frequencies may be attenuated in a scattering corona around the neutron star. We find that upper limits to the unattenuated power of some of the strongest theoretically predicted harmonics and sidebands are as low as $\sim 2$ per cent of the unattenuated power of the high-frequency QPO in Sco X-1.
\end{abstract}

Key words: accretion, accretion discs - stars: individual: Sco X-1 - stars: oscillations stars: neutron $-\mathrm{X}$-rays: stars.

\section{INTRODUCTION}

It is four years now since the kilohertz quasi-periodic oscillations ( $\mathrm{kHz}$ QPOs) were discovered in the persistent flux of Scorpius X-1 (van der Klis et al. 1996a) and 4U 1728-34 (Strohmayer, Zhang \& Swank 1996). In the meantime, similar kHz QPOs have been seen in some 20 other low-mass X-ray binaries (LMXBs; see van der Klis 2000 for a review). These QPOs often appear in pairs, with frequencies $\nu_{1}$ and $\nu_{2}\left(\nu_{2}>\nu_{1}\right)$ between $\sim 400$ and $\sim 1300 \mathrm{~Hz}$, which in a given source can shift by a few hundred $\mathrm{Hz}$, apparently as a function of mass accretion rate.

Most of the models proposed so far assume that one of the $\mathrm{kHz}$ QPOs reflects the Keplerian orbital motion at some preferred radius in the accretion disc (e.g., Miller, Lamb \& Psaltis 1998; Stella \& Vietri 1999; Osherovich \& Titarchuk 1999), but there are other explanations as well (Klein et al. 1996a,b; Jernigan, Klein \& Arons 2000). In recent discussions (Lamb \& Miller 1999; Stella 1999; Psaltis 1999) it was emphasized that empirical discrimination between two of the leading classes of models is possible in principle by studying the harmonic and sideband structure of the $\mathrm{kHz}$ QPOs. Here we concentrate only on these two model classes

In the 'sonic-point' model (SPM; Miller et al. 1998), the QPO at $\nu_{2}$ (the upper QPO) is produced at the radius where the radial flow velocity in the disc turns from subsonic to supersonic (the sonic radius), and the QPO at $\nu_{1}$ (the lower QPO) originates by a beat between the upper QPO and the spin frequency of the neutron star. In the 'relativistic-precession' model (RPM; Stella \& Vietri 1999)

${ }^{\star}$ E-mail: mmendez@fcaglp.unlp.edu.ar the QPO at $\nu_{2}$ is also assumed to be Keplerian, but the QPO at $\nu_{1}$ is produced by the apsidal precession of a slightly non-circular inner accretion disc. The QPO frequencies in the RPM are calculated for test particles in purely geodesic relativistic motion, i.e. neglecting the hydrodynamical and radiative effects of the accretion flow. However, Psaltis \& Norman (2000) have recently proposed a dynamical model in which the QPOs are produced by oscillations in the accretion disk. In this model, which we will call 'transition-radius' model (TRM), there is a transition radius in the accretion disc that acts as a band-pass filter with resonances near the orbital and periastron-precession frequencies.

Besides the main peaks at $\nu_{1}$ and $\nu_{2}$, the SPM and the TRM predict other (weaker) harmonics and sidebands of these QPOs, at specific frequencies. For instance, the SPM predicts a relatively strong harmonic of the lower QPO at $2 \nu_{1}$ (see table 3 of Miller et al. 1998 for a list of other sideband peaks predicted by the SPM), whereas the TRM predicts a sideband at $2 \nu_{2}-\nu_{1}$ (cf. equation (29) in Psaltis \& Norman 2000). In principle, the detection of a QPO at $2 \nu_{1}$ and a non-detection of a QPO at $2 \nu_{2}-\nu_{1}$ would tend to rule out the TRM, whereas the detection of a QPO at $2 \nu_{2}-\nu_{1}$ and a non-detection of a QPO at $2 \nu_{1}$ would tend to rule out the SPM (Miller 2000).

In this paper, we use data from the Rossi X-ray Timing Explorer $(R X T E)$ to search for the predicted harmonics and sidebands in the power spectrum of the $\mathrm{kHz}$ QPO source Sco X-1. Because of its very high flux, Sco X-1 has extremely significant $\mathrm{kHz}$ QPOs, and hence a very sensitive study of any harmonic structure is possible. We do not detect any of these secondary QPOs. The fact that we detect the $\mathrm{kHz}$ QPOs at $\nu_{1}$ and $\nu_{2}$, but none of these other peaks sets severe constraints on the models currently proposed to explain the $\mathrm{kHz}$ QPOs in LMXBs. 


\section{OBSERVATIONS}

We used data from the Proportional Counter Array (PCA; Jahoda et al. 1996) on board RXTE (Bradt, Rothschild \& Swank 1993) taken on 1996 February 14, 18, 19, 1996 May 24-28, 1997 March 15, 1997 April 18-24, 1997 August 22, 1998 January 2-8, 1998 February 27, 28, 1998 May 30, 31, 1998 June 1, 2, 1998 July 2-5, 1999 January 6, 8-11 and 13-16. Each observation (i.e. each part of the data with a unique $R X T E$ ID number) consists of data blocks of $\sim 60$ to $\sim 3700$ s interrupted by passages of the satellite through the South Atlantic Anomaly and occultations of the source by the Earth. The total usable time was $\sim 630 \mathrm{ks}$.

To avoid detector safety triggers, telemetry saturation, and to reduce the dead-time effects produced by the high count rate of Sco X-1, some observations were carried out with the source slightly off-axis, with some of the five proportional counter units of the PCA switched off, recording only photons detected by the upper anode chain of the PCA, recording only photons from a limited energy range, or using a combination of these constraints. In all cases, high-time resolution data were available with a time resolution of $0.25 \mathrm{~ms}$ or better. For our analysis below we combined all the available data, irrespective of whether they were collected using any of the above observational constraints. When single and double-event data were recorded in parallel (see van der Klis et al. 1996b), we combined them off-line to enhance the sensitivity.

\section{ANALYSIS AND RESULTS}

We divided the high time resolution data into 16-s segments, and produced a power spectrum for each of these segments up to a Nyquist frequency of $2048 \mathrm{~Hz}$ (for $\sim 50$ per cent of the data we also produced power spectra up to a Nyquist frequency of $4096 \mathrm{~Hz}$ ). For 69 per cent of the observations the power spectra were calculated using the full PCA energy band. For the rest of the power spectra we used data from selected energy bands (24 per cent of the power spectra were calculated between 5 and $18 \mathrm{keV}$, 5 per cent between 5 and $60 \mathrm{keV}$, and 2 per cent between 2 and $18 \mathrm{keV}$ ) because we found that the $\mathrm{kHz}$ QPOs were more significant in those energy bands, or because those were the only energy bands available. Finally, we averaged together groups of 8 contiguous 16-s power spectra to produce average power spectra.

We searched these average power spectra for $\mathrm{kHz}$ QPOs, at frequencies $\gtrsim 250 \mathrm{~Hz}$. We did this by first identifying those power spectra that showed a strong QPO, and visually estimating its frequency, $\nu_{0}$. When two QPOs were present in the power spectra, we always picked the one at higher frequency. It turned out that in the cases where only one $\mathrm{kHz}$ QPO was visible, it was always the upper $\mathrm{kHz}$ QPO (see below). We then fitted the power spectra, in the range $\nu_{0}-100$ to $\nu_{0}+100 \mathrm{~Hz}$, using a function consisting of a constant, a power law and one Lorentzian. We discarded the power spectra for which the QPOs were less than $3 \sigma$ significant. We note that in this manner we might have discarded data with weak QPOs that could have been detected averaging together more data. There were 1384 average power spectra with significant $\mathrm{kHz}$ QPOs, equivalent to $177152 \mathrm{~s}$ of data.

For those observations where we detected only one QPO in the average power spectra we applied the 'shift-and-add' technique (Méndez et al. 1998a) to try and detect the second QPO: based on the frequency of the detected QPO, we aligned and averaged all the power spectra of a single observation. In those cases, this procedure revealed a second $\mathrm{kHz} \mathrm{QPO}$, which in all cases was at a lower frequency than the QPO peak that we used to align the power spectra, showing that all the initial frequency measurements in the 1384 average power spectra corresponded to the upper $\mathrm{kHz}$ QPO.

Next, we measured the frequency separation between the $\mathrm{kHz}$ QPOs as a function of the upper QPO frequency, $\nu_{2}$. We proceeded as follows: we aligned the 1384 power spectra using the upper $\mathrm{kHz}$ QPO as a reference; we grouped the data in 49 sets, each of them containing $\sim 40-\sim 210$ power spectra, such that $\nu_{2}$ did not vary by more than $5-10 \mathrm{~Hz}$ within each set, and we combined these aligned spectra to produce an average power spectrum for each set. We fitted these 49 power spectra in the range $400-1300 \mathrm{~Hz}$ using a function consisting of a constant, a power law and two Lorentzians. The fits were good, with reduced $\chi^{2} \leq 1.1$ for 279 degrees of freedom, and the significance of both peaks was always $>3 \sigma$.

Fig. 1 shows that $\Delta \nu=\nu_{2}-\nu_{1}$, the frequency difference between the two QPOs, decreases from $\sim 310$ to $\sim 240 \mathrm{~Hz}$, as $\nu_{2}$ increases from $\sim 840$ to $\sim 1100 \mathrm{~Hz}$. This figure can be compared to fig. 3a of van der Klis et al. (1997), who first reported the decrease in $\Delta \nu$ with $\nu_{2}$ in Sco X-1. Because we included more data, and because we used the shift-and-add technique to measure $\Delta \nu$, the errors are smaller in the present figure than in the previous one, and some structure, particularly between 900 and $1020 \mathrm{~Hz}$, becomes apparent (perhaps this structure is related to the 'bump' seen in the plot of $\Delta \nu$ versus $\nu_{1}$ of $4 \mathrm{U} 1608-52$ at $\nu_{1} \sim 700 \mathrm{~Hz}$; see fig. 3 in Méndez et al. 1998b).

From Fig. 1 we can read off $\nu_{1}$ as a function of $\nu_{2}$; because we already know $\nu_{2}$ for each average power spectrum, we can calculate the expected frequencies of hypothetical signals at multiples of $\nu_{1}$ and $\nu_{2}$, or at frequencies that are combinations of these two frequencies, for each power spectrum. As we described in Section 1, some of these frequencies are important in the context of the models proposed to explain the $\mathrm{kHz}$ QPOs.

We calculated the following quantities: $2 \nu_{1}, 2 \nu_{2}, \nu_{2}-\nu_{1}$, $\nu_{2}+\nu_{1}, 2 \nu_{1}-\nu_{2}, 2 \nu_{2}-\nu_{1}, 2 \nu_{1}+\nu_{2}, 2 \nu_{2}+\nu_{1}$ and $2\left(\nu_{2}-\nu_{1}\right)$. We then aligned, in turn, the power spectra on each of the above

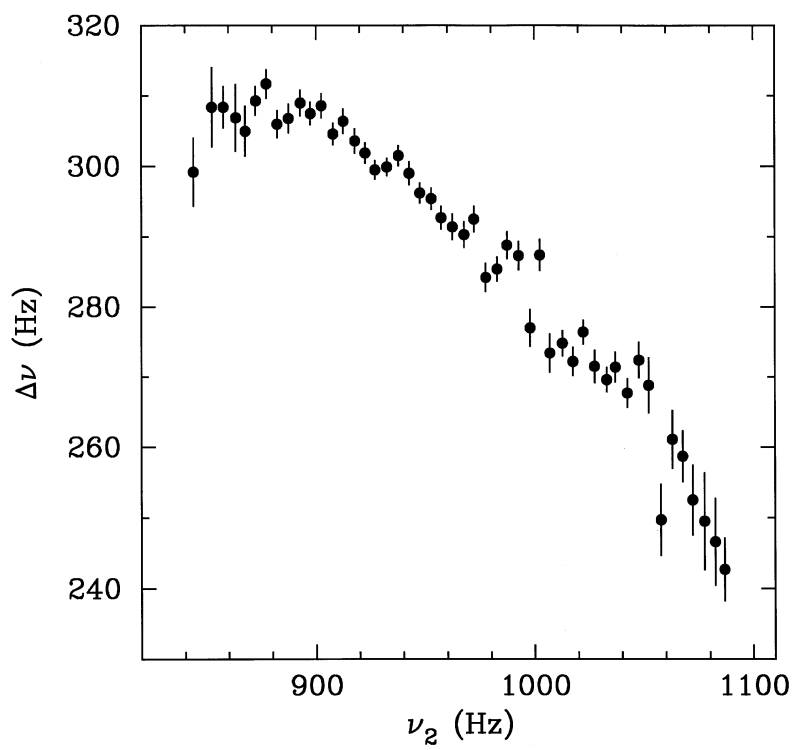

Figure 1. The frequency separation between the $\mathrm{kHz}$ QPOs in Sco X-1 as a function of the upper $\mathrm{kHz}$ QPO frequency. 


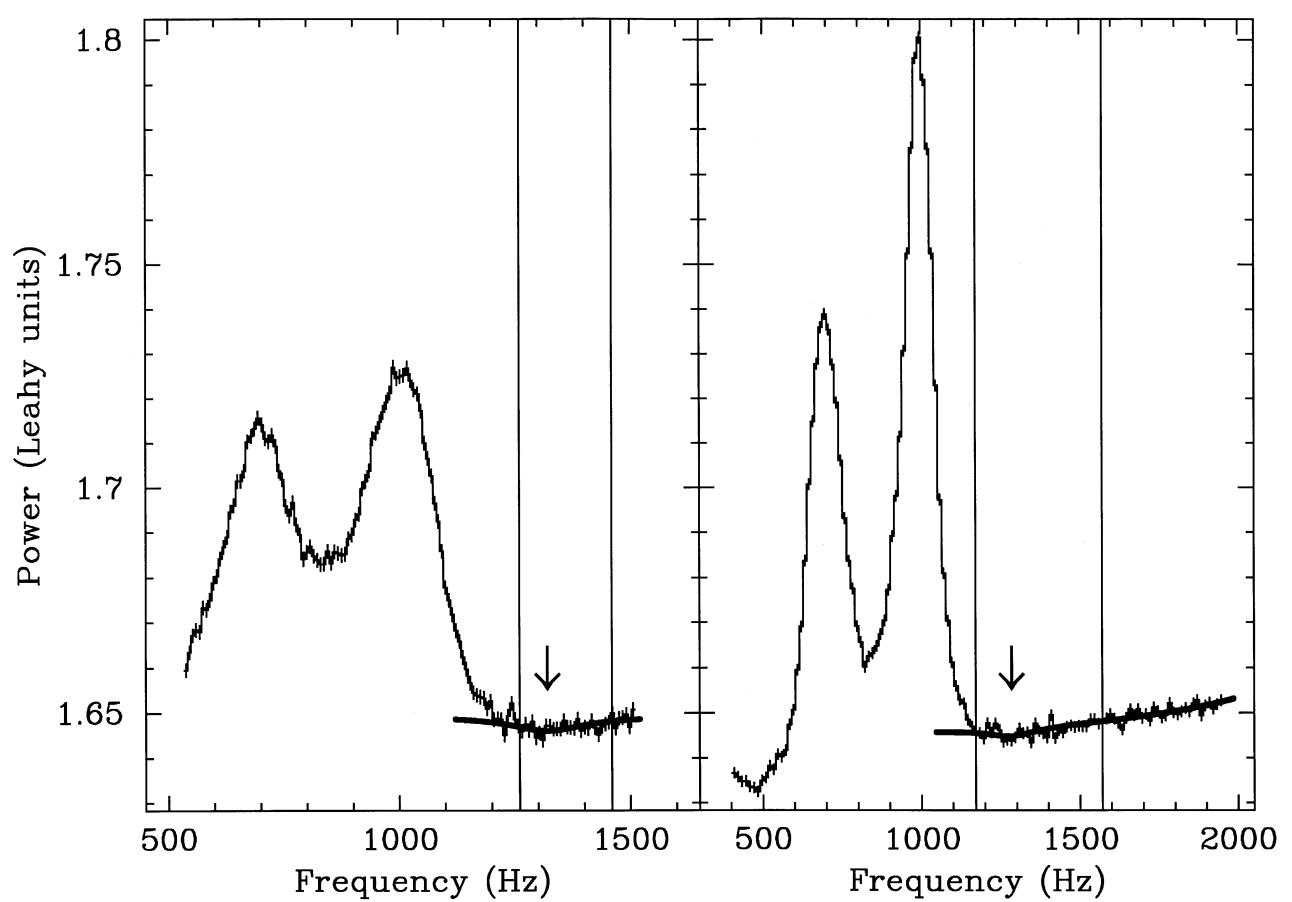

Figure 2. Shifted-and-averaged power spectra of Sco X-1. The average power spectrum shown in the left panel was computed by first aligning the individual power spectra such that a QPO peak at $2 \nu_{1}$ would always end up at $1320 \mathrm{~Hz}$, indicated by the arrow. In the right panel a QPO peak at $2 \nu_{2}-\nu_{1}$ would similarly end up at $1284 \mathrm{~Hz}$ (arrow). In both panels, the vertical lines show the frequency range used to fit the data (see text). Notice that the frequency alignment also altered the apparent shapes and frequencies of the strong QPO peaks.

frequencies, and averaged them together to try and detect a signal present at any of these frequencies. We fitted these shifted and averaged power spectra in a segment of $\sim 400 \mathrm{~Hz}$ that included the expected frequency, using a constant plus a Lorentzian with the centroid fixed in each case at the expected frequency, and with a fixed FWHM of $200 \mathrm{~Hz}$, approximately equal to the sum of the FWHM of each of the QPOs (van der Klis et al. 1996b). This value is about two times larger than the largest FWHM measured in the QPOs of this and other sources, and yields conservative upper limits to the unobserved harmonics and sidebands; tighter upper limits could be obtained by fixing the FWHM to a smaller value. No detections result from choosing a smaller FWHM in the current data set. In a few cases we added a power law to the fit to take account of a sloping continuum. The shape of the power spectrum at high frequencies is dominated by dead-time effects (Zhang et al. 1995) which, in the case of Sco X-1 at a count rate that exceeds $\sim 25000 \mathrm{cs}^{-1} \mathrm{PCU}^{-1}$, are large and not yet sufficiently well understood to predict the shape of the highfrequency part of the power spectrum accurately. We note that a better knowledge of this effect could yield tighter upper limits than those that we report in this paper.

We did not detect a significant QPO at any of the above frequencies. As an example, in Fig. 2 we show two power spectra that were shifted to $2 \nu_{1}$ and to $2 \nu_{2}-\nu_{1}$, the frequencies of the main high-frequency peaks (besides the $\mathrm{kHz}$ QPOs) predicted by the models of Miller et al. (1998) and Psaltis \& Norman (2000), respectively.

Upper limits are shown in Table 1. To calculate these upper limits we varied the amplitude of the Lorentzian until the $\chi^{2}$ of the fit increased by 2.71 with respect to the best-fitting value ( 95 per cent confidence level for a single parameter); the upper limits we quote in Table 1 represent the change in the amplitude for this change in the $\chi^{2}$. Because of the observational constraints
Table 1. Upper limits to the observed amplitudes of the harmonics and sidebands of the $\mathrm{kHz}$ QPOs in Sco X-1

\begin{tabular}{lcc}
\hline Frequency & Predicted frequency range $(\mathrm{Hz})$ & Upper limit $^{a}$ \\
\hline $2 \nu_{1}$ & $1090-1690$ & 0.12 \\
$2 \nu_{2}$ & $1690-2170$ & 0.13 \\
$\nu_{2}-\nu_{1}$ & $240-300$ & 0.30 \\
$\nu_{2}+\nu_{1}$ & $1390-1930$ & 0.08 \\
$2 \nu_{1}-\nu_{2}$ & $240-600$ & 0.26 \\
$2 \nu_{2}-\nu_{1}$ & $1140-1330$ & 0.12 \\
$2 \nu_{1}+\nu_{2}$ & $1930-2770$ & 0.10 \\
$2 \nu_{2}+\nu_{1}$ & $2230-3020$ & 0.10 \\
$2\left(\nu_{2}-\nu_{1}\right)$ & $480-600$ & 0.21 \\
\hline
\end{tabular}

${ }^{a} 95$ per cent confidence upper limits to the rms fractional amplitude of the QPO at the indicated frequency, in units of the observed rms fractional amplitude of the QPO at $\nu_{2}$. This QPO has an amplitude between 0.6 and 2.5 per cent (van der Klis et al. 1997).

described in Section 2, and because we combined power spectra computed from data in different energy bands, we cannot give upper limits in units of fractional rms amplitude (van der Klis 1995); instead, all the upper limits in Table 1 are in units of the observed fractional rms amplitude of the QPO at $\nu_{2}$. Any peaks at the predicted frequencies are between 11 and 156 times weaker than the peak at $\nu_{2}$, which translates into rms amplitude ratios between 0.08 and 0.30 .

\section{DISCUSSION}

We have measured the frequencies of the two simultaneous $\mathrm{kHz}$ QPOs, $\nu_{1}$ and $\nu_{2}$, in Sco X-1 in a large data set. We have used these measurements to calculate the expected frequencies of hypothetical signals at harmonics and sidebands of these QPOs, as 
Table 2. Upper limits to the unattenuated amplitude of the harmonics and sidebands of the $\mathrm{kHz}$ QPOs in Sco X-1

\begin{tabular}{lcccccc}
\hline Frequency & $\tau^{a}$ & $R(\mathrm{~km})^{a}$ & Upper limit $^{b}$ & $\tau^{a}$ & $R(\mathrm{~km})^{a}$ & Upper limit $^{c}$ \\
\hline $2 \nu_{1}$ & $<1$ & 336 & 0.15 & 8.7 & 129 & 0.30 \\
$2 \nu_{2}$ & 6.5 & 202 & 0.27 & 9.8 & 109 & 0.93 \\
$\nu_{2}-\nu_{1}$ & $<1$ & $<10$ & 0.30 & $<1$ & $<10$ & 0.30 \\
$\nu_{2}+\nu_{1}$ & 5.7 & 262 & 0.11 & 9.1 & 121 & 0.35 \\
$2 \nu_{1}-\nu_{2}$ & $<1$ & $<10$ & 0.26 & $<1$ & $<10$ & 0.26 \\
$2 \nu_{2}-\nu_{1}$ & $<1$ & 352 & 0.14 & 8.3 & 138 & 0.23 \\
$2 \nu_{1}+\nu_{2}$ & 7.3 & 167 & 0.37 & 9.8 & 109 & 1.43 \\
$2 \nu_{2}+\nu_{1}$ & 7.8 & 151 & 0.54 & 10.2 & 103 & 2.16 \\
$2\left(\nu_{2}-\nu_{1}\right)$ & $<1$ & $<10$ & 0.21 & $<1$ & $<10$ & 0.21 \\
\hline
\end{tabular}

${ }^{a}$ Optical depth and radius of a possible scattering cloud around Sco X-1, obtained by minimizing equation (3) at each frequency (see text). These are the values that were used to calculate the corresponding upper limits shown in this table.

${ }^{b}$ Upper limits for the unattenuated amplitude of the QPO at the indicated frequency for a luminosity oscillation (see equation (1)), in units of the unattenuated amplitude of the QPO at $\nu_{2}$.

${ }^{c}$ Upper limits for the unattenuated amplitude of the QPO at the indicated frequency for a beaming oscillation (see equation (2)), in units of the unattenuated amplitude of the QPO at $\nu_{2}$. In both cases the QPO at $\nu_{2}$ is assumed to be a beaming oscillation (Miller et al. 1998).

predicted by two classes of $\mathrm{kHz}$ QPO models. We used a sensitive technique to search for these harmonics and sidebands in the power spectra of Sco X-1, but none was detected. Their power is between 10 and 100 times less than the power of the upper $\mathrm{kHz}$ QPO (95 per cent confidence).

Oscillations produced close to the surface of the neutron star can be attenuated in a scattering corona. The attenuation is generally larger for oscillations produced by a pencil beam sweeping the surroundings (beaming oscillations), than for oscillations due to actual changes of the luminosity with time (luminosity oscillations; Brainerd \& Lamb 1987; Kylafis \& Klimis 1987; Kylafis \& Phinney 1989; Miller et al. 1989). The attenuation factor (the ratio of the amplitude $A_{\infty}$ of the oscillations at infinity to their original amplitude $A_{0}$ ) as a function of frequency $\nu$, for a scattering corona of a radius $R$ and optical depth $\tau$ is approximately given by:

$\frac{A_{\infty, \text { lum }}}{A_{0, \text { lum }}}=2^{3 / 2} x \mathrm{e}^{-x}+\mathrm{e}^{-\tau}$

for a luminosity oscillation, and by

$\frac{A_{\infty, \text { beam }}}{A_{0, \text { beam }}}=\frac{2^{5 / 2} x \mathrm{e}^{-x}}{1+\tau}+\mathrm{e}^{-\tau}$,

for a beaming oscillation, with $x=(3 \pi \nu R \tau / c)^{1 / 2}$ (Kylafis \& Phinney 1989).

We can use these relations to calculate the largest unattenuated amplitudes allowed by the data in Table 1. Because at each frequency the upper limit on the ratio of observed amplitudes is $A_{\infty, y}(\nu) / A_{\infty, \text { beam }}\left(\nu_{2}\right)$ (Table 1 ), the upper limit on the ratio of unattenuated amplitudes $A_{0, y}(\nu) / A_{0 \text {, beam }}\left(\nu_{2}\right)$, can be obtained by minimizing

$\alpha_{y}(\nu)=\frac{A_{\infty, y}(\nu) / A_{0, y}(\nu)}{A_{\infty, \text { beam }}\left(\nu_{2}\right) / A_{0, \text { beam }}\left(\nu_{2}\right)}$

with respect to $\tau$ and $R$, with the additional constraint that $A_{\infty, \text { beam }}\left(\nu_{2}\right) / A_{0, \text { beam }}\left(\nu_{2}\right)>0.01$ (because the QPO at $\nu_{2}$ is detected at an amplitude $\gtrsim 1$ per cent; van der Klis et al. 1997). In equation (3), $y$ stands either for luminosity or beaming (see equations (1) and (2)), and we have assumed that the QPO at $\nu_{2}$ is a beaming oscillation (Miller et al. 1998).

The upper limits to the unattenuated amplitudes of the sidebands and harmonics of the $\mathrm{kHz}$ QPOs, both for luminosity and beaming oscillations, are shown in Table 2 . In units of the amplitude of the QPO at $\nu_{2}$, the amplitudes of these harmonics and sidebands range from 0.11 to 0.54 for a luminosity oscillation, and from 0.21 to 2.16 for a beaming oscillation (assuming beaming for the QPO at $\nu_{2}$ ). From the minimization of $\alpha_{y}(\nu)$ at the frequencies listed in Table 2, we get that optical depths $\tau \sim$ $1-10$ and radii $R \sim 1-350 \mathrm{~km}$ for the putative scattering cloud in Sco X-1 would be most effective at suppressing the sidebands and harmonics. These values are comparable to those obtained by Vaughan et al. (1997, 1998), $\tau \lesssim 5$ and $R \lesssim 80 \mathrm{~km}$, for 4U $1608-$ 52 from measurements of the time lags of the $\mathrm{kHz}$ QPOs (see also Kaaret et al. 1999). We want to mention that the upper limits given in Table 2 have been calculated assuming properties of the scattering corona that maximize the attenuation at each frequency independently. In reality, if the corona exists it would have fixed values of $\tau$ and $R$, so that if it is effective at suppressing the oscillations at some frequencies, it will be less effective at others.

In conclusion, there is a remarkable lack of harmonic and sideband structure in the $\mathrm{kHz}$ QPOs of Sco X-1. None of the frequencies predicted by the two main classes of models are detected, with upper limits implying they are one to two orders of magnitude weaker in power than the main peaks. This sets severe constraints on either the purity of the $\mathrm{kHz}$ QPO generation mechanism in Sco X-1, or the parameters of the scattering corona that surrounds the neutron star.

\section{ACKNOWLEDGMENTS}

We would like to thank various participants of the 1999 workshop on X-ray Probes of Relativistic Astrophysics at the Aspen Center for Physics for pleasant and extremely fruitful discussions. We are specially grateful to Coleman Miller, Fred Lamb, Luigi Stella, and Dimitrios Psaltis. We thank Coleman Miller, Fred Lamb and Tomaso Belloni for comments that helped us to improve the original manuscript. MM acknowledges comments from Mikhail Revnivtsev concerning the dead-time effects of the PCA. This 
work was supported by the Netherlands Research School for Astronomy (NOVA), the Netherlands Organization for Scientific Research (NWO) under contract number 614-51-002 and the NWO Spinoza grant 08-0 to E. P. J. van den Heuvel. MM is a fellow of the Consejo Nacional de Investigaciones Científicas y Técnicas de la República Argentina. This research has made use of data obtained through the High Energy Astrophysics Science Archive Research Center Online Service, provided by the NASA/ Goddard Space Flight Center.

\section{REFERENCES}

Bradt H. V., Rothschild R. E., Swank J. H., 1993, A\&AS, 97, 355

Brainerd J., Lamb F. K., 1987, ApJ, 317, L33

Jahoda K., Swank J. H., Giles A. B., Stark M. J., Strohmayer T., Zhang W., Morgan E. H., 1996, Proc. SPIE 2808: EUV, X-ray, and Gamma-ray Instrumentation for Astronomy VII, p. 59

Jernigan J. G., Klein R. I., Arons J., 2000, ApJ, 530, 875

Kaaret P., Piraino S., Ford E. C., Santangelo A., 1999, ApJ, 514, L31

Klein R. I., Arons J., Jernigan J. G., Hsu J., 1996a, ApJ, 457, L85

Klein R. I., Jernigan J. G., Arons J., Morgan E. H., Zhang W., 1996b, ApJ, 469, L119

Kylafis N. D., Klimis G. S., 1987, ApJ, 323, 678

Kylafis N. D., Phinney E. S., 1989, in Ögelman H., van den Heuvel E. P. J., eds, Timing Neutron Stars, NATO ASI Series C262, p. 27

Lamb F. K., Miller M. C., 1999, paper presented at the 1999 Aspen Summer Workshop on X-ray Probes of Relativistic Astrophysics
Méndez M. et al., 1998a, ApJ, 494, L65

Méndez M., van der Klis M., Wijnands R., Ford E. C., van Paradijs J., Vaughan B. A., 1998b, ApJ, 505, L23

Miller M. C., 2000, in Proc. of the Bologna Conf. X-ray Astronomy 1999: Stellar Endpoints, AGN, and the Diffuse X-ray Background, in press (astro-ph/0007287)

Miller M. C., Lamb F. K., Psaltis D., 1989, ApJ, 791

Osherovich V., Titarchuk L., 1999, ApJ, 522, L113

Psaltis D., 1999, paper presented at the 1999 Aspen Summer Workshop on $\mathrm{X}$-ray Probes of Relativistic Astrophysics

Psaltis D.,Norman C., 2000, ApJ, in press (astro-ph/0001391)

Stella L., 1999, paper presented at the 1999 Aspen Summer Workshop on X-ray Probes of Relativistic Astrophysics

Stella L., Vietri M., 1999, PRL, 82, 17

Strohmayer T., Zhang W., Swank J., 1996, IAU Circ. 6320,

van der Klis M., 1995, in Lewin W. H. G., van Paradijs J., van den Heuvel E. P. J., eds, X-ray Binaries. Cambridge Univ. Press, Cambridge, p. 252 van der Klis M., 2000, ARA\&A, in press (astro-ph/0001167)

van der Klis M., Swank J., Zhang W., Jahoda K., Morgan E., Lewin W., Vaughan B., van Paradijs J., 1996a, IAU Circ. 6319,

van der Klis M., Swank J. H., Zhang W., Jahoda K., Morgan E. H., Lewin W. H. G., Vaughan B., van Paradijs J., 1996b, ApJ, 469, L1

van der Klis M., Wijnands R. A. D., Horne K., Chen W., 1997, ApJ, 481, L97 Vaughan B. A. et al., 1997, ApJ, 483, L115

Vaughan B. A. et al., 1998, ApJ, 509, L145

Zhang W., Jahoda K., Swank J. H., Morgan E. H., Giles A. B., 1995, ApJ, 449,930

This paper has been typeset from a $\mathrm{T}_{\mathrm{E}} \mathrm{X} / \mathrm{L} \mathrm{T}_{\mathrm{E}} \mathrm{X}$ file prepared by the author. 